\title{
Impacto da utilização de recursos tecnológicos no desempenho de atividades cotidianas e na satisfação da mãe de criança com paralisia cerebral grave
}

\section{The impact of the use of technological resources on the performance daily activities and the satisfaction of the mother having child with severe cerebral palsy}

\author{
Rafaela Brigoni Barbosa ${ }^{1}$, Mariana Dutra Zafani ${ }^{2}$, Luciana Ramos Baleotti ${ }^{3}$
}

http://dx.doi.org/10.11606/issn.2238-6149.v30i1p19-26

Barbosa RB, Zafani MD, Baleotti LR. Impacto da utilização de recursos tecnológicos no desempenho de atividades cotidianas e na satisfação da mãe de criança com paralisia cerebral grave. Rev Ter Ocup Univ São Paulo. 2019 jan.-abr.;30(1):19-26.

RESUMO: O objetivo geral deste estudo foi o de identificar a percepção da mãe de uma criança com paralisia cerebral quadriplégica espástica grave, com três anos de idade, sobre os recursos tecnológicos utilizados por seu filho no desempenho de tarefas funcionais da rotina diária. Os objetivos específicos foram: listar e indicar a frequência de modificações utilizadas pela criança de acordo com o Inventário de Avaliação Pediátrica (PEDI); identificar o nível de satisfação da mãe em relação as modificações, as dificuldades para usá-las e a necessidade de orientações. Para a coleta de dados utilizou-se o PEDI e um roteiro de entrevista semiestruturado. Os resultados sugerem que a mãe sentia-se satisfeita com os recursos tecnológicos utilizados por seu filho e estava bem instruída em relação ao modo de usá-los. Dentre os aspectos negativos que influenciavam na usabilidade dos recursos, citam-se à falta de acessibilidade das vias públicas para o uso da cadeira de rodas e a baixa renda da família. Os resultados deste estudo reforçam a necessidade de envolver a família no processo de seleção de recursos tecnológicos que exigem assistência máxima do cuidador, e reportam para a relevância de o profissional analisar criteriosamente o contexto ambiental em que eles serão inseridos a fim de facilitar a usabilidade e promover, de fato, uma melhor qualidade de vida para a pessoa com deficiência e seus familiares.

DESCRITORES: Equipamentos de autoajuda; Paralisia cerebral; Percepção.
Barbosa RB, Zafani MD, Baleotti LR. The impact of the use of technological resources on the performance daily activities and the satisfaction of the mother having child with severe cerebral palsy. Rev Ter Ocup Univ São Paulo. 2019 Jan.-Apr.;30(1):19-26.

ABSTRACT: The general objective of this study was to identify how the mother of a three-year-old child with severe spastic quadriplegic cerebral palsy perceives the technological resources used by her child to perform daily functional tasks. The specific objectives were: list and indicate how often, according to the Pediatric Assessment Inventory (PEDI), the child used modifications; identify the level of satisfaction of the mother regarding such modifications, the difficulties to apply them and the need for guidelines. For data collections, we used the PEDI and a semi-structured interview script. The results suggest that the mother was pleased with the technological resources used by her son and well advised about how to apply them. Among the negative aspects influencing the usability of such resources, this work found public places lacking wheelchair accessibility and low family income. While the results of this study reinforce the need to engage the family in the process of choosing the technological resources, they also show how relevant it is to have a professional who may consistently analyze the environmental context in which such resources are found and so allow for an easier use and indeed promote a better quality of life for the disabled person and their family members.

KEYWORDS: Self-help devices; Cerebral palsy; Perception.

Trabalho Oral apresentado no VIII Encontro Científico de Terapia Ocupacional, Faculdade de Filosofia e Ciências, UNESP, Marília, dez. 2017. 1. Terapeuta Ocupacional, Aprimoramento e Especialização em Reabilitação e Tecnologia, Faculdade de Filosofia e Ciências, UNESP, Campus de Marília, São Paulo, Brasil. ORCID: 0000-0002-0158-3231. Email: rafa_brigoni@hotmail.com.

2. Terapeuta Ocupacional. Doutora em Educação pela Faculdade de Filosofia e Ciências, UNESP, Campus de Marília, São Paulo, Brasil. ORCID: 0000-0003-2875-6021. Email: mari_dzafani@hotmail.com.

3. Professora Assistente Doutora - nível II. Docente do curso de Terapia Ocupacional do Departamento de Fisioterapia e Terapia Ocupacional, Faculdade de Filosofia e Ciências, UNESP, Campus de Marília, São Paulo, Brasil. Docente do Programa de Pós-Graduação em Desenvolvimento Humano e Tecnologias, Instituto de Biociências, UNESP, Campus de Rio Claro. ORCID: 0000-002-3300-2075. Email: baleotti@marilia.unesp.br. Endereço para correspondência: Luciana Ramos Baleotti. Avenida Hygino Muzzi Filho, 737. Bairro: Mirante. Marília, SP, Brasil. CEP: 17.525-900. Email: baleotti@marilia.unesp.br. 


\section{INTRODUÇÃO}

s alterações nas estruturas e funções corporais
que acometem as crianças com paralisia cerebral grave podem trazer impactos para o desempenho de atividades cotidianas e interferir na qualidade de vida. Tais alterações somadas às inadequações ambientais podem restringir a participação nos diferentes contextos de vida ${ }^{1}$.

Quanto mais comprometida do ponto de vista global for a criança, maiores serão as dificuldades na realização de atividades o que irá requerer uso abrangente de recursos de Tecnologia Assistiva (TA), que podem potencializar o desempenho em atividades. A TA abarca recursos, produtos, metodologias, estratégias, serviços e práticas capaz de contribuir de maneira grandiosa para a promoção da funcionalidade e participação de pessoas com deficiência, mobilidade reduzida ou incapacidade ${ }^{2}$.

Estudos demonstraram que as expectativas em relação ao uso de TA, manifestadas por cuidadoras de crianças com paralisia cerebral grave, por um lado, referiam-se ao desejo de que as crianças melhorassem a autoestima, adquirissem autonomia, habilidades sociais e escolares. Por outro lado, demonstraram insegurança quanto à capacidade das crianças no uso do recurso e a possibilidade de frustração das expectativas familiares ${ }^{3}$.

Além da utilização de recursos de TA, podem ocorrer comprometimentos severos nas funções orgânicas, as quais irão demandar por exemplo, a substituição da função alimentar ou gastrointestinal por meio de procedimentos médicos extensivos, exigindo assistência máxima do cuidador nas tarefas diárias. Pais de crianças com paralisia cerebral relataram sobre as reações emocionais negativas que sentiram devido a introdução artificial de métodos de alimentação ${ }^{4}$. Outro estudo identificou que a maior parte das famílias entrevistadas aceitaram o procedimento da gastrostomia após a indicação médica. Relacionaram esse achado à existência do trabalho multidisciplinar e concluíram que pais que recebem melhores informações, podem aceitar e compreender melhor a indicação da gastrostomia ${ }^{5}$.

É importante que as equipes profissionais da reabilitação forneçam orientações sobre a condição de saúde da criança, sobre os recursos de TA e outros recursos tecnológicos utilizados no ambiente domiciliar. Crianças gravemente comprometidas, normalmente, fazem uso de diferentes recursos para o desenvolvimento das mais variadas atividades.

Há reconhecimento generalizado de que os serviços especializados destinados ao atendimento de crianças com deficiência necessitam contar com a participação e colaboração de suas famílias. A participação da família no processo de intervenção direcionado à criança, por meio das orientações que lhe são dadas pelos diferentes profissionais, e a postura de acolhimento e escuta dos profissionais em relação aos sentimentos da família precisam ser tratadas como parte integrante de qualquer atendimento destinado a crianças com deficiência ${ }^{6}$.

Dentre os benefícios decorrentes da parceria entre a família e os profissionais, pode-se citar: credibilidade no processo terapêutico ${ }^{7}$, melhoria dos vínculos ${ }^{8}$, exercício mais satisfatório e seguro dos cuidados prestados pela família à criança ${ }^{8,9,10}$, extensão de ações da terapia para casa $^{7}$, criação compartilhada de estratégias para a resolução das dificuldades?

Assim, é relevante a escuta atenciosa em relação às dificuldades manifestadas pela família no cuidado diário à criança e no uso dos diferentes recursos que lhe são prescritos. É preciso entender o sentido das tecnologias para a família. Interroga-se se, de fato, as tecnologias propiciam a melhora da condição de vida. Nessa perspectiva, o foco não recai sobre o recurso tecnológico em si, mas sim, na experiência vivenciada pela família na interlocução com as tecnologias e o efeito delas no cotidiano. No entanto, essa temática tem sido explorada de maneira insuficiente junto aos familiares de crianças gravemente comprometidas.

Frente a essas considerações, este estudo de caso visa contribuir para o entendimento do uso de diferentes recursos tecnológicos na percepção da mãe. Teve como objetivo geral identificar a percepção da mãe de uma criança com paralisia cerebral grave sobre os recursos tecnológicos utilizados por seu filho no desempenho de tarefas funcionais de autocuidado, mobilidade e de função social. Os objetivos específicos foram: listar e indicar a frequência de modificações utilizadas pela criança de acordo com o Inventário de Avaliação Pediátrica (PEDI); identificar o nível de satisfação da mãe em relação as modificações utilizadas, as dificuldades para usá-las e a necessidade de orientações advindas dos profissionais.

\section{PROCEDIMENTOS METODOLÓGICOS}

Este estudo recebeu a aprovação do Comitê de Ética em Pesquisa da Faculdade de Filosofia e Ciências, Unesp, Marília, sob parecer $\mathrm{N}^{\circ}$ 0957/2007, em conformidade com a Resolução 196/96, atendendo aos preceitos da ética na pesquisa envolvendo seres humanos.

Esta pesquisa caracteriza-se como estudo de caso único, com abordagem descritiva e qualitativa. Por ser estudo de caso único, adotou-se como critério de inclusão a seleção de uma criança com grave comprometimento 
funcional do ponto de vista global. Pois, crianças com paralisia cerebral, semelhantes a do presente estudo, podem necessitar de recursos de TA e de outros recursos tecnológicos para o desenvolvimento das mais variadas atividades cotidianas.

A mãe da criança tinha 34 anos de idade, cursou o Ensino Médio completo e era casada. A criança do sexo masculino, com três anos e três meses de idade, diagnóstico clínico de paralisia cerebral grave do tipo quadriplégica espástica, causada por infecção viral (toxoplasmose congênita), associada a anoxia perinatal. Quanto ao nível da função motora grossa, de acordo com o Gross Motor Function Classification System $(G M F C S)^{11}$, a criança enquadrava-se no nível V, sendo este classificado como o mais grave do ponto de vista de função motora grossa, o que indica que a que a criança necessita de cadeira de rodas com extensas adaptações. Apesentava também baixa visão, alterações da fala e da deglutição. Necessitava de assistência total da cuidadora para a realização de todas as atividades cotidianas e não frequentava escola.

A criança era atendida, desde os seis meses de idade, no Centro de Estudos da Educação e Saúde (CEES), uma clínica escola que oferece assistência ambulatorial nas áreas de fisioterapia, fonoaudiologia e terapia ocupacional a sujeitos com deficiências diversas. Esta clínica escola é uma unidade auxiliar da Faculdade de Filosofia e Ciências UNESP - Campus de Marília.

Para a coleta de dados foram utilizados o Inventário de Avaliação Pediátrica de Incapacidade (PEDI) e um Roteiro de Entrevista semiestruturado. O PEDI avalia a capacidade funcional de crianças de seis meses a sete anos e meio de idade, traduzido, adaptado e validado para o contexto brasileiro por Mancini ${ }^{12}$. O PEDI é dividido em três partes. A parte I informa sobre as habilidades funcionais da criança para realizar atividades e tarefas de seu cotidiano, nas áreas de autocuidado, mobilidade e função social. A parte II informa sobre a independência da criança, documentada pela quantidade de ajuda fornecida pelo cuidador principal, na realização de tarefas funcionais nas mesmas áreas citadas. E, por fim, a parte III do PEDI documenta a quantidade de atividades funcionais nas áreas citadas para as quais são necessárias modificações.

Neste estudo, foi utilizada a parte III do PEDI em função das características de total dependência da criança para a realização de atividades cotidianas, o que sugere a necessidade de modificações, ou seja, a utilização de recursos tecnológicos para o desempenho de atividades. A parte III constitui-se em categoria nominal que indica a frequência de modificações em cada categoria definida pelo PEDI, sendo: nenhuma modificação (NM), quando a criança não faz uso de nenhuma modificação para o desempenho da tarefa funcional. Modificação centrada na criança (MCC), quando a modificação ou modificações utilizadas forem aquelas que normalmente são utilizadas por crianças com desenvolvimento típico, como, por exemplo, pentes, talheres em tamanho infantil. Modificação de reabilitação (MR), quando a criança utiliza equipamento destinado para crianças com deficiências tais como cadeira de rodas, pranchas de transferência. Modificação extensiva (ME), quando a modificação ou modificações envolverem mudanças arquitetônicas, roupas feitas sob medida, uso de cateteres para controle da bexiga, gastrostomia, entre outros.

O Roteiro de Entrevista foi desenvolvido especificamente para este estudo, elaborado de acordo com as categorias presentes no PEDI: autocuidado, mobilidade e função social. Para cada categoria, visavase a obtenção das seguintes informações: satisfação em relação as modificações usadas pela criança, nesse item a cuidadora escolheu uma das três alternativas de resposta, sendo: satisfeita, pouco satisfeita e insatisfeita, e, em seguida, justificou o motivo da sua escolha; dificuldades na utilização das modificações e a necessidade de orientações quanto ao seu uso.

A coleta de dados foi realizada no CEES no mês de junho de 2017, em um único dia, de acordo com data e horário previamente agendados com a mãe, por meio de entrevista, com duração aproximada de 60 minutos. As entrevistas foram gravadas em áudio e posteriormente transcritas.

Para a análise de dados do PEDI, foi verificada a frequência das alternativas de resposta dada a cada categoria definida pela parte III do PEDI, posteriormente, foi feita análise descritiva dos dados. Quanto ao roteiro de entrevista, este foi analisado qualitativamente a partir da leitura cuidadosa e atenta dos relatos o que possibilitou identificar e organizar as unidades de análise, as quais se constituíram por trechos de falas inseridos nas categorias de autocuidado, mobilidade e função social.

\section{RESULTADOS}

Primeiramente, são apresentados os dados relativos à análise da frequência de modificações em cada uma das áreas do PEDI, cabe relembrar que a criança requeria assistência total da mãe para a realização de todas as atividades cotidianas. Tais dados são apresentados nas tabelas subsequentes. 
Barbosa RB, et al. Impacto da utilização de recursos tecnológicos. Rev Ter. Ocup Univ São Paulo. 2019 jan./abr.;30(1):19-26.

Tabela 1 - Frequência de modificações na área de autocuidado

\begin{tabular}{lcccc}
\hline Itens & NM & MCC & MR & ME \\
\hline Alimentação & 0 & 0 & 0 & 1 \\
Higiene Pessoal & 1 & 0 & 0 & 0 \\
Banho & 0 & 0 & 1 & 0 \\
Vestir - parte superior do corpo & 1 & 0 & 0 & 0 \\
Vestir - parte inferior do corpo & 1 & 0 & 0 & 0 \\
Banheiro & 1 & 0 & 0 & 0 \\
Controle urinário & 1 & 0 & 0 & 0 \\
Controle intestinal & 0 & 1 & 0 & 0 \\
Frequência & 5 & 1 & 1 & 1 \\
\hline
\end{tabular}

Fonte: Elaborada pelas autoras.

Legenda: NM- Nenhuma modificação; MCC - Modificação centrada na criança; MR - Modificação de reabilitação; ME - modificação extensiva.

Conforme Tabela 1, a criança fazia uso de três recursos na área de autocuidado. O primeiro recurso (controle intestinal) referia-se ao redutor de assento infantil para vaso sanitário, este foi inserido na subcategoria MCC. $\mathrm{O}$ segundo recurso foi inserido na subcategoria $\mathrm{ME}$, pois tratava-se da utilização de sonda gástrica para alimentação, utilizada em virtude de a criança apresentar problemas de deglutição. E, por fim, um recurso de TA (subcategoria MR) sendo banheira adaptada, com apoio de cabeça, material antiderrapante, alta em relação ao solo para favorecer o alinhamento biomecânico e a postura ortostática da mãe no momento do banho da criança. A banheira, embora adequada para a mãe, estava totalmente incompatível com a altura e peso da criança.

$\mathrm{Na}$ Tabela 2, apresentam-se os dados relativos à existência de modificações na área da mobilidade.

Tabela 2 - Frequência de modificações na área de mobilidade

\begin{tabular}{lcccc}
\hline Itens & NM & MCC & MR & ME \\
\hline $\begin{array}{l}\text { Transferências no banheiro/ } \\
\text { cadeiras }\end{array}$ & 1 & 0 & 0 & 0 \\
$\begin{array}{l}\text { Transferências no carro/ônibus } \\
\text { Mobilidade na cama/ }\end{array}$ & 0 & 1 & 0 & 0 \\
transferência & 1 & 0 & 0 & 0 \\
Transferências no chuveiro & 1 & 0 & 0 & 0 \\
Locomoção em ambiente interno & 0 & 0 & 1 & 0 \\
Locomoção em ambiente externo & 0 & 0 & 1 & 0 \\
Escadas & 1 & 0 & 0 & 0 \\
Frequência & 4 & 1 & 2 & 0 \\
\hline
\end{tabular}

Fonte: Elaborada pelas autoras.

Legenda: NM - Nenhuma modificação; MCC - Modificação centrada na criança; MR - Modificação de reabilitação; ME - modificação extensiva.
A mãe informou que a MCC correspondia a uma cadeira infantil para automóvel, utilizada para acomodar a criança ao ser transportada no carro da família. A MR referia-se a outro recurso de TA, sendo a cadeira de rodas adaptada. Na tabela acima a frequência de MR é 2, devido ao fato de a mesma cadeira de rodas ser utilizada tanto em ambiente interno como externo. Para os demais itens, não foram relatadas a existência de modificações.

Quanto a área da função social, a criança dependia totalmente da mãe para o desempenho de todos os aspectos, que englobam compreensão e expressão funcional, resolução de problemas em parceria, brincar com companheiro e segurança. Todas as respostas foram inseridas na subcategoria NM.

Na sequência, apresentam-se os dados advindos do roteiro de entrevista, explicitados por categorias de acordo com o PEDI.

\section{Categoria 1 - Autocuidado}

Com relação as modificações utilizadas na área de autocuidado, a mãe informou estar satisfeita com o uso do redutor de assento e da sonda gástrica para alimentação. Relatou que não tinha dificuldades e não necessitava de orientações para o uso de tais recursos pois recebeu orientações dos profissionais que atendiam a criança. $\mathrm{O}$ trecho abaixo exemplifica a opinião da mãe quanto a sonda gástrica:

Eu não tenho dúvidas sobre o uso e nem dificuldade com a sonda, só precisa de cuidado para limpar, mas eu já fui orientada e estou acostumada. A única coisa que eu tenho que ficar atenta é com as crianças, pois elas têm curiosidade de mexer no botão que regula a velocidade, e ai se pingar muito rápido o D. vomita.

Com relação ao redutor de assento, destaca-se que embora a criança não tenha adquirido o controle de esfíncter anal e vesical, a mãe relatou que a criança evacuava normalmente nos mesmos horários. Assim, a colocava no vaso sanitário na tentativa de ela aprender a controlar o esfíncter e indicar a necessidade do uso do banheiro.

No que se refere a banheira adaptada, a mãe destacou sentir-se pouco satisfeita. No entanto, tal sentimento não tinha relação com as características da banheira, mas sim, com o fato de a mesma estar pequena para a criança, conforme o relato abaixo:

Com a banheira adaptada é bem mais fácil de dar banho. A banheira já está pequena, mas é uma banheira cara, então eu espero que ela aguente mais um ano, pois eu não tenho como comprar outra nesse momento. 
Cabe destacar que a mãe apontou não ter dificuldade e não precisar de orientações para o uso da banheira, pois foi orientada pela terapeuta ocupacional no momento da aquisição.

\section{Categoria 2 - Mobilidade}

No que se refere as modificações utilizadas na área da mobilidade, que engloba transferências e mobilidade em ambientes internos e externos, a mãe relatou estar satisfeita com o uso da cadeira infantil utilizada para transferência no carro. Informou não apresentar dificuldade e não necessitar de orientações quanto ao seu uso, pois já havia recebido orientações previamente.

Com relação a cadeira de rodas, a mãe referiu que estava pouco satisfeita. Quando questionada sobre o motivo de sua insatisfação informou que o recurso atendia as necessidades da criança, porém a presença de barreiras arquitetônicas em ambiente domiciliar e externo dificultavam o seu uso. O peso corporal do filho também impactou na satisfação da mãe, pois as transferências posturais eram realizadas por ela, e a criança estava grande e pesada. Os trechos exemplificam esses aspectos:

Tenho dificuldade para levar o D. para o quarto, pois a cadeira de rodas não passa pela porta, então eu tenho que levá-lo no colo, e ele está muito pesado. Eu que faço tudo para ele porque meu marido trabalha fora, então eu que tenho que cuidar, levar para o médico, atendimentos, tudo.

Tem lugar que não está preparado para receber uma criança que usa cadeira de rodas. As calçadas são cheias de buracos e D. acaba "sacudindo" muito, e tem muitos lugares que tem escadas e não tem elevador, então eu tenho que pegá-lo no colo, e o peso dificulta muito.

E, por fim, a mãe informou ter sido orientada pelos profissionais da fisioterapia e da terapia ocupacional quanto as transferências posturais e o manuseio da cadeira de rodas, não sentindo assim a necessidade de mais orientações.

\section{Categoria 3 - Função Social}

Conforme mencionado anteriormente, a criança não utilizava modificações no desempenho de tarefas funcionais na área de função social. No entanto, destacase que apresentava manifestações comportamentais que eram interpretadas pela mãe como forma de expressão e comunicação social, conforme ilustrado no trecho seguinte:
Eu acho que ele me entende, porque quando eu pergunto alguma coisa, ele resmunga. O pai e o irmão também conseguem entender. Nós temos o costume de sair todos os dias no mesmo horário e quando o horário de sair na rua se aproxima, D. fica inquieto e só se acalma quando a gente sai de casa. Mas já aconteceu de ele chorar bastante, eu fiquei preocupada, mas não conseguia saber o que ele tinha, aí levei ao médico e ele estava com infecção na garganta.

\section{DISCUSSÃO}

Em relação ao uso de recursos de TA constatou-se a utilização de dois recursos, inseridos na categoria de MR (banheira e cadeira de rodas adaptadas). Possivelmente com avanço da idade e, consequentemente, com a alteração e ampliação dos papéis ocupacionais da criança, novos recursos tecnológicos e de TA serão necessários a fim de promover sua participação em diferentes contextos e tarefas funcionais. $\mathrm{O}$ ingresso da criança na escola, por exemplo, poderá demandar modificações nos recursos didático-pedagógicos utilizados por ela, nas edificações, no mobiliário escolar, no currículo, dentre outras. Normalmente, crianças com paralisia cerebral gravemente comprometidas necessitam de recursos que ampliem uma habilidade funcional deficitária ou possibilitem "[...] a realização da função desejada e que se encontra impedida por circunstância de deficiência [...] (p. 2)"'13. Tendo em vista a gravidade do quadro clínico apresentado pela criança deste estudo, uma análise criteriosa e multiprofissional será essencial para a prescrição de recursos e equipamentos que atendam suas características e necessidades motoras, perceptivas, de comunicação e sensoriais.

Ainda no que se refere ao uso de recursos de TA, verificou-se que na área da função social a criança não fazia uso de nenhum recurso, porém a mãe relatou que a família buscava desenvolver estratégias para compreender as manifestações dos desejos da criança, mas nem sempre isso era possível. Por meio do choro da criança, a mãe percebia que algo estava errado, porém não conseguia definir o que era. Situações como essas podem ser angustiantes tanto para a criança quanto para a família. Diante disso, recursos que favoreçam o potencial comunicativo da criança podem ser positivos para sua participação social e relação familiar. A Comunicação Suplementar e/ou Alternativa pode ser uma estratégia bem sucedida nesse caso, trata-se de uma área da ciência voltada às necessidades comunicativas de pessoas com impedimentos ou dificuldades ao desenvolvimento da oralidade ${ }^{14}$.

Das cinco modificações utilizadas pela criança, a mãe referiu satisfação com três delas (cadeira infantil 
(MCC), redutor de assento (MCC) e sonda gástrica (ME), não apresentar dificuldades para utilizá-las e não necessitar de orientações referentes ao uso das mesmas. Essas opiniões indicam a adequação dos recursos às necessidades da criança e da família. Além disso, dão indícios de que a família estava assistida pelos profissionais que acompanhavam a criança.

Ter um filho com condições de saúde bastante frágeis, como é o caso da criança estudada, nos remete à reflexão sobre a importância de os profissionais da saúde munir a família de conhecimento e habilidades específicas que lhes possibilite dar atenção de qualidade para a criança, sem medo ou angústias. É possível perceber que a mãe foi orientada adequadamente em relação aos cuidados que deveria ter com a sonda gástrica e, parece que ela apresentava sentimento de tranquilidade e confiança para manuseá-la ao dizer: "[...] precisa de cuidado para limpar, mas eu já estou acostumada, já aprendi. [...]”. Os profissionais da saúde detêm o conhecimento necessário para auxiliar a família a realizar os cuidados ao filho com deficiência, principalmente quando teme pela saúde dele e se questiona sobre o que fazer e como fazer para assisti-lo ${ }^{15}$.

Com relação ao redutor de assento, pode-se inferir que a mãe tinha a expectativa de que seu filho adquirisse o controle esfincteriano e independência para o uso do banheiro. No entanto, de acordo com as condições de saúde da criança, sabe-se que o seu prognóstico é desfavorável para tal independência.

Os profissionais que prestam serviços de reabilitação devem compreender a percepção das mães e dos demais familiares frente à condição da deficiência a fim de desenvolverem ações que vão além da técnica e, que visem o suporte psicológico e a postura de acolhimento e escuta em relação aos sentimentos da famíliai ${ }^{16,17}$. Milbrath et al. ${ }^{18}$ enfatizaram a importância de os profissionais da saúde adotarem uma linguagem precisa e clara para se comunicar com a família, e a necessidade de demostrarem receptividade para esclarecer as dúvidas da família e de fazer um feedback a fim de verificar o nível de compreensão a respeito das informações fornecidas. Os mesmos autores apontaram para a importância de a comunicação se dar de "[...] forma horizontal, levando em conta que todos os comunicantes são considerados sujeitos" (p. 733) ${ }^{18}$.

Esses aspectos podem colaborar para que a família crie mecanismos psíquicos para elaborar emocionalmente o fato de ter um filho gravemente comprometido, e adquira condições emocionais que diminuam e/ou eliminem o sentimento de negação frente à gravidade, a fim de compreenderem a realidade que se apresenta.

A banheira e a cadeira de rodas adaptadas referem-se as modificações que a mãe relatou estar pouco satisfeita. No que diz respeito a banheira, a mãe apontou não ter dificuldade para o uso desse recurso e não precisar de orientações, porém referiu estar pouco satisfeita com o seu uso. A opinião da mãe indica que este recurso facilitava a atividade de banho, possivelmente por favorecer a sua postura corporal, diminuindo a sobrecarga física. Recursos de TA podem tanto favorecer o desempenho autônomo e independente em tarefas cotidianas como facilitar o cuidado de pessoas em situação de dependência de auxílio ${ }^{13}$. No entanto, a usabilidade do recurso estava prejudicada, pois a banheira estava pequena para a criança e a mãe não tinha condição financeira para adquirir uma nova, interferindo negativamente na sua satisfação.

Quanto a cadeira de rodas, a mãe referiu estar pouco satisfeita, sobretudo, em função de barreiras arquitetônicas presentes tanto no ambiente domiciliar quanto externo e também devido ao peso do filho, o que dificultava a realização das transferências posturais.

A queixa da mãe com relação ao peso corporal do filho requer atenção especial dos profissionais da saúde. Orientações em relação às técnicas adequadas para fazer mudanças posturais são importantes, assim como recomendações visando a prevenção de dores na coluna. Nesse sentido, Alpino et al. ${ }^{19}$ realizaram intervenção junto a mães de adolescentes com paralisia cerebral que envolveu orientações e treinamento de habilidades específicas sobre cuidados com os filhos e consigo próprias (exercícios de auto alongamento, relaxamento, percepção corporal). Os resultados desse estudo revelaram que todas as mães avaliaram positivamente a intervenção, além disso, identificou-se melhora das habilidades relacionadas aos cuidados dos filhos, com persistência de dificuldades em algumas manobras terapêuticas, atividades de transferências e banho.

O relato da mãe referente as barreiras arquitetônicas é outro elemento importante e precisa ser considerado. Nem sempre a família tem condições financeiras para realizar modificações arquitetônicas em sua residência, como é o caso da participante deste estudo. Além disso, a literatura aponta que normalmente as mães são as cuidadoras primárias do filho com deficiência, como ocorre com a mãe do presente estudo, e isso pode dificultar a inserção delas no trabalho formal e, consequentemente, impedir a colaboração financeira com as despesas domiciliares. Almeida et al. ${ }^{17} \mathrm{em}$ um estudo realizado com mães de crianças com paralisia cerebral constataram que, em muitos casos, as mães abandonaram o trabalho e/ou o estudo a fim de prestarem cuidados ao filho, o que acarretava em sobrecarga financeira em função de gastos adicionais com reabilitação, medicamentos, adaptações na estrutura física da casa, entre outros. Esse estudo apontou também as dificuldades relativas à falta de acessibilidade decorrente das condições precárias das ruas e da falta de acesso aos locais públicos. 
A falta de acessibilidade em espaços de uso comum mencionado pela mãe deste estudo, bem como na literatura, trata-se de um problema público evidenciado em nosso país. Uma das principais dificuldades relatadas por pais de crianças com deficiência física relaciona-se ao trajeto de casa até a escola devido à presença de buracos nas calçadas, bem como calçadas estreitas, com diversas barreiras como lixeiras, telefone público e pontos de ônibus mal colocados ${ }^{20,21}$.

A acessibilidade arquitetônica em ambientes de uso comum deve ser priorizada pelos gestores municipais a fim de que o acesso seja irrestrito e possibilite a participação e inclusão social para toda e qualquer pessoa, independente de sua condição física, sensorial, de idade, social e outras.

\section{CONCLUSÕES}

Neste estudo, buscou-se compreender a percepção da mãe sobre os recursos tecnológicos utilizados por seu filho para apoio nas atividades de autocuidado, mobilidade e função social, pois essa percepção, tanto negativa quanto positiva, pode trazer impactos para a qualidade de vida do cuidador primário, no caso deste estudo, a mãe.

Os resultados apontaram que o nível socioeconômico desfavorecido interferiu na aquisição de recurso de TA (banheira adaptada) adequado para a criança, bem como na possibilidade da realização de mudanças arquitetônicas no ambiente domiciliar. Circunstâncias dessa natureza podem estar presentes no cotidiano de muitas famílias de crianças com deficiência, e remete à reflexão sobre a importância dos sistemas governamentais proverem condições que atendam às necessidades dessas famílias e tornem o cotidiano delas menos oneroso.

Outro elemento mencionado pela mãe refere-se à falta de acessibilidade em ambientes externos e de uso comum. Atualmente ainda se constata a inadequação em ambientes diversificados, a falta de acesso ao transporte público, entre outros, isso é consequência, em parte, de políticas públicas que não favorecem e/ou não priorizam alternativas que busquem tornar os ambientes acessíveis para todos. Nesse sentido, torna-se relevante que os profissionais da saúde priorizem ações junto aos familiares, no sentido de oferecer subsídios que ampliem o conhecimento sobre as políticas públicas nacionais de acessibilidade a fim de fortalecê-los e promover o empoderamento para que busquem os seus direitos.

É desejável a continuidade desta investigação com a ampliação do número de participantes a fim de verificar se os resultados encontrados representam uma tendência comum do que ocorre com a percepção de mães referente aos recursos utilizados por crianças com paralisia cerebral com limitações funcionais globais acentuadas.

Contribuições dos autores: Barbosa RB. -foi responsável pela coleta e análise de dados e discussão dos resultados; Zafani MD e Baleotti $L R$ - foram responsáveis pela concepção do estudo, análise e discussão dos resultados e revisão e redação final do texto.

Autoria e indicação de responsabilidades: As autoras trabalharam na concepção, no delineamento, na análise e interpretação dos dados, na redação do artigo e na aprovação da versão a ser publicada.

\section{REFERÊNCIAS}

1. Baleotti LR, Santos LA, Zafani MD. Avaliação de habilidades motoras de uma criança com paralisia cerebral incluída em contexto escolar regular. Rev Educ Espec. 2015;28:149-64. doi: http://dx.doi.org/10.5902/1984686X13503.

2. Brasil. Secretaria Especial dos Direitos Humanos. Subsecretaria Nacional de Promoção dos Direitos da Pessoa com Deficiência. Comitê de Ajudas Técnicas. Tecnologia assistiva. Brasília: CORDE; 2009. Disponível em: https:// www.pessoacomdeficiencia.gov.br/app/sites/default/files/ publicacoes/livro-tecnologia-assistiva.pdf.

3. Bittencourt ZZLC, Cheraid DC, Montilha RCI, Gasparetto MERF. Expectativas quanto ao uso de tecnologia assistiva. J Res Spec Educ Needs. 2016;16:492-6. doi: https://doi. org/10.1111/1471-3802.12311.
4. Susin FP, Bortolini V, Sukiennik R, Mancopes R, Barbosa L De Rosa. Perfil de pacientes com paralisia cerebral em uso de gastrostomia e efeito nos cuidadores. Rev CEFAC. 2012;14(5):933-42. doi: http://dx.doi.org/10.1590/S151618462012005000016.

5. Rouse L, Herrington P, Assey J, Baker R, Golden S. Feeding problems, gastrostomy and families: a qualitative pilot study. Brit J Learning Disabil. 2002;30(3):122-8. doi: 10.1046/j.14683156.2002.00149.x

6. Baleotti LR, Omote S, Gregorutti CC. Oficina de atividades: espaço de atenção aos familiares de crianças com deficiência. Psicol Estud. 2015;20(1):3-12. doi: http://dx.doi.org/10.4025/ psicolestud.v20i1.23507. 
Barbosa RB, et al. Impacto da utilização de recursos tecnológicos. Rev Ter. Ocup Univ São Paulo. 2019 jan./abr.;30(1):19-26.

7. Givigi R, Santos A, Ramos G. Um novo olhar sobre participação da família no processo terapêutico. Rev Ter Ocup Univ São Paulo. 2011;22(3):221-8. doi: https://doi.org/10.11606/ issn.2238-6149.v22i3p221-228.

8. Dantas MSA. Atenção profissional à criança com paralisia cerebral e sua família. Rev Enferm UERJ. 2017;25:1-6. doi: http://dx.doi.org/10.12957/reuerj.2017.18331.

9. Travassos-Rodriguez F, Carneiro-Féres T. Os bebês com síndrome de Down e seus pais: novas propostas para intervenção. Estud Psicol. 2012;29:831-40. Disponível em: http://www.scielo.br/pdf/estpsi/v29s1/19.pdf.

10. Almeida PS, Gonçalves TP, Maciel D.G. Paralisia cerebral: dificuldades apresentadas pelas mães no enfrentamento do diagnóstico, no segmento do tratamento fisioterapêutico em casa e nos cuidados diários. REBES Rev Bras Educ Saúde. 2014;4(4):19-28. Disponível em: https://www.gvaa.com.br/ revista/index.php/REBES/article/view/2633/2595.

11. Palisano RJ, et al. Gross motor function classification system for cerebral palsy. Dev Med Child Neurol. 1997;39:214-23.

12. Mancini MC. Inventário de avaliação pediátrica de incapacidade (PEDI). Manual da versão brasileira adaptada de: Pediatric evaluation of disability inventory (PEDI). Belo Horizonte: UFMG; 2005. p.193.

13. Bersch R. Introdução à tecnologia assistiva. Porto Alegre: Centro Especializado em Desenvolvimento Infantil; 2008 [citado 17 fev. 2018]. Disponível em: http://www.soplaar.com/ material_individual/pdf/144S832O4P507L538A401R111.pdf.

14. Ferreira-Donati GC, Deliberato D. Questionário de necessidades de informação em linguagem e comunicação alternativa
(QNILCA-F) - versão para família. Rev Bras Educ Espec. 2017;23(1):53-66. doi: 10.1590/s1413-65382317000100005.

15. Barbosa MAM, Pettengill MAM, Farias TL, Lemes LC. Cuidado da criança com deficiência: suporte social acessado pelas mães. Rev Gaucha Enferm (porto Alegre). 2009;3:406-12. Disponível em: https://seer.ufrgs.br/RevistaGauchadeEnfermagem/article/ view/8224/6962.

16. Baleotti LR, Omote S. A concepção de deficiência em discussão: ponto de vista dos docentes de Terapia Ocupacional. Cad Bras Ter Ocup. 2014;22(1):71-8. doi: http://dx.doi.org/10.4322/ cto.2014.008.

17. Almeida TCS, Ruedell AM, NobrevJRS, Tavares KO. Paralisia cerebral: Impacto no cotidiano familiar. Rev Bras Cien Saúde. 2015;19(3):171-8. doi: 10.4034/RBCS.2015.19.03.01.

18. Milbrath VM, Siqueira HCH, Motta MGC, Amestoy SC. Comunicação entre a equipe de saúde e a família da criança com asfixia perinatal grave. Texto Contexto Enferm. 2011;20(4):726-34. doi: 10.1590/S0104-07072011000400011.

19. Alpino MAS, Valenciano PJ, Furlaneto BB, Zechim FC. Orientações de Fisioterapia a mães de adolescentes com paralisia cerebral: abordagem educativa para o cuidar. Rev Bras Educ Espec. 2013;19(4):597-610. doi: 10.1590/ S1413-65382013000400009.

20. Canotilho MM. A integração de crianças portadoras de deficiência física no ensino regular segundo a perspectiva de seus pais. Rev Bras Educ Espec. 2002;8(1):15-26.

21. Pereira LMF, Caribé D, Guimarães P, Matsuda D. Acessibilidade e crianças com paralisia cerebral: a visão do cuidador primário. Fisioter Mov. 2011;24(2):299-306. doi: 10.1590/S0103-51502011000200011. 